\title{
Front-line staff's perspective on patient safety culture in Iranian medical centers: A systematic review and meta-analysis
}

$\underline{\text { Khosravizadeh, Omid }}\left|\underline{\text { Mohseni, Mohammad }}{ }^{\mathrm{b}}\right|$ Baghian, Najmeh $^{\mathrm{c}}\left|\underline{\text { Maleki, Aisa }}{ }^{\mathrm{d}}\right| \underline{\text { Hashtroodi, }}$ Ailard $^{\mathrm{d}}$ | Yari, Saeed ${ }^{\mathrm{e} ; \mathrm{f}}$

[a] Social Determinants of Health Research Center, Research Institute for Prevention of NonCommunicable Diseases, Qazvin University of Medical Sciences, Qazvin, Iran

[b] Health Management and Economics Research Center, Iran University of Medical Sciences, Tehran, Iran

[c] Research Development Center, Shahid Rahnemoon Hospital, Shahid Sadoughi University of Medical Sciences, Yazd, Iran

[d] Student Research Committee, Qazvin University of Medical Sciences, Qazvin, Iran

[e] Student Research Committee, Department of Health, Shahid Beheshti University of Medical Sciences, Tehran, Iran

[f] Department of Occupational Health Engineering, Faculty of Health, Neyshabur University of Medical Sciences, Neyshabur, Iran

Correspondence: [*] Address for correspondence: Aisa Maleki, Student Research Committee, Qazvin University of Medical Sciences, Qazvin, Iran. Tel.: +98 9223695917; Email: AisaMalekii@gmail.com

Abstract: BACKGROUND: The prerequisite for promoting safety culture is to assess the existing safety culture level of institutes, because safety precautions without appropriate evaluation increase costs and unforeseen risks. OBJECTIVE: This study aimed to systematically review the status of patient safety culture from the perspective of clinical personnel at Iranian hospitals through a meta-analysis of studies using the Hospital Survey on Patient Safety Culture (HSOPSC) questionnaire. METHODS: The present systematic review and meta-analysis was conducted in 2018. Data were gathered by searching Google Scholar, Scopus, PubMed, and Web of Science databases up to November 2018. Search keywords were "patient", "safety", "culture", "healthcare", "hospital", "medical center", "HSOPSC tool", and "Iran". The search protocol was limited to 10 years. To estimate the PSC score, computer software CMA:2 (Comprehensive MetaAnalysis) was used. The presence of heterogeneity across the studies was assessed with the I2 statistic. A forest plot was used to report the results. Publication bias was assessed through a funnel plot. RESULTS: The meta-analysis of studies showed that the PSC score based on the random effect model was 52.7\% (95\% CI: 50.2\%-55.2\%), $(\mathrm{Q}=522.3$, df $=54, \mathrm{P}<0.05, \mathrm{I} 2=89.6)$. A mean of 12 dimensions of HSOPSC showed that the "Teamwork within units" dimension had the highest PSC score (67.2\%) and "Non-punitive response to error" had the lowest score (40.4\%). CONCLUSIONS: Managers and policymakers should be directed towards non-punitive responses to errors and persuade staff to report errors and execute the approach to learn from mistakes. Also, a periodic government evaluation of the patient safety culture will help further its sustainable development.

Keywords: Patient safety, safety culture, HSOPSC, medical staff, Iran DOI: $10.3233 /$ JRS-191021 